ORIGINAL ARTICLE

\title{
Assessment of Level of Patient Satisfaction after Prostatectomy for Benign Prostatic Hyperplasia in Referral Hospitals in Addis Ababa.
}

\author{
Andualem Deneke ${ }^{1 *}$, Mezgeb Gedefe ${ }^{2}$
}

\footnotetext{
OPEN ACCESS

Citation: Andualem Deneke, Mezgeb Gedefe. Assessment of Level of Patient Satisfaction after Prostatectomy for Benign Prostatic Hyperplasia at Tikur Anbesa Specialized Hospital and Menilik II Referral Hospitals in the Period of August $1 / 2017$ to June 15/2018. Ethiop J Health Sci.2020;30(5):733. doi:http:// dx.doi.org/ 10.4314/ejhs.v30i5.12

Received: May 3, 2020

Accepted: May 25, 2020

Published: September 1, 2020

Copyright: (C) 2020 Andualem Deneke, et

al. This is an open access article distributed under the terms of the Creative Commons Attribution License, which permits unrestricted use, distribution, and reproduction in any medium, provided the original author and source are credited. Funding: Addis Ababa University Competing Interests: The authors declare that this manuscript was approved by all authors in its form and that no competing interest exists.

Affiliation and Correspondence:

${ }^{1}$ Associate Professor of Surgery,

Consultant in Urology, Addis Ababa

University, College of Health

Sciences, Ethiopia

${ }^{2}$ Urologist, Menilik II Hospital;

Addis Ababa Ethiopia

*Email: andualemdb94@yahoo.com
}

\begin{abstract}
BACKGROUND: Benign Prostatic hyperplasia is a very common problem in aging men. TURP comprises $95 \%$ of all surgical procedures and is the treatment of choice for prostates sized between $30 \mathrm{ml}$ and $80-100 \mathrm{ml}$. Open surgery is the treatment of choice for large glands (bigger than 80-100ml) and for those with associated complications that have indication for open surgery. Many literatures show that the overall patient satisfaction rate and clinical outcome of TURP for BPH are very good. The objective of this study was 'assessing the level of patient satisfaction after undergoing TURP and TVP for $B P H$.

METHODS: In this research, convenient sampling technique was used. The study design was prospective cohort study. Standardized questioner was prepared in English and later translated into Amharic. Information about demographic characteristics, preoperative data, intraoperative data, and immediate postoperative data were taken while the patient was in the hospital. At the first and the third months after the prostatectomy, patients were inquired on their level of satisfaction about their disease specific satisfaction on the urinary function and their sexual function. We used HCAHPS Measure of Patient Satisfaction Tool, to collect data and analyzed using SPSS version 18.

RESULTS: A total of 89 patients were enrolled in the study among which $65.2 \%$ had undergone TURP. The rate of major perioperative complication was found to be low. Over half of the patients had postoperative hospital stay of three or less days. Majority of the patients were satisfied with the care given by the nurses (68.2\%) and doctors (84.3\%) with relatively higher physicians' care satisfaction level. More than $60 \%$ of the patients were highly satisfied with their urinary surgical outcome. With regard to hospital environment, around $60 \%$ of the patients reported that they were very satisfied with cleanliness of the rooms, bath rooms and the quietness of the rooms at night.

CONCLUSIONS: Both TURP and TVP have high level of patient satisfaction associated with low perioperative major complications. Therefore both can be recommended for patients with clear indications for prostatectomy.

KEYWORDS: Prostate, hyperplasia, Prostatectomy, Satisfaction
\end{abstract}




\section{INTRODUCTION}

Benign Prostatic Hyperplasia (BPH) is a very common problem in aging men. Based on different literatures, BPH with troublesome lower urinary tract symptoms can affect around 30\% of the men aged greater than 65 years (1). According to standard treatment guidelines, $\mathrm{BPH}$ can be managed with many treatment modalities based on the level of symptomatology and/or disease progression (e.g. expectant management, medical treatment, minimally invasive treatments, Transurethral Resection of the Prostate (TURP), open prostatectomy etc) $(1,2)$. In the best set ups, TURP comprises $95 \%$ of all surgical procedures and is the treatment of choice for prostates sized between $30 \mathrm{ml}$ and $80-100 \mathrm{ml}$ (1). Open surgery is the treatment of choice for large glands (greater than 80-100 ml), and for those with associated complications such as large bladder stones, or bladder diverticula which needs resection (1).

Many literatures show that the overall patient satisfaction rate and clinical outcome of TURP for $\mathrm{BPH}$ are very good (see literature review). However, researches that directly assess the level of satisfaction after open prostatectomy are very few. In this study, we tried to show to what level patients were satisfied after the commonly performed procedure (open prostatectomy) in resource limited setups like ours, and that of TURP which is considered to be gold standard for management of $\mathrm{BPH}$.

Patient satisfaction has a variety of definitions in different literatures, but most definitions have 'underlying patient expectation' as a common variable. One of the literatures defines it as;

Patient satisfaction is a cognitive evaluation and an emotional reaction to medical care that is strongly influenced by underlying expectations (3).

Defining and consequently assessing the level of patient's satisfaction is quite difficult. As such, patient-experience does not simply reflect clinical outcomes or adherence-driven outcomes, rather it seeks to represent a unique encompassing dimension that is challenging to measure objectively (4). The goal of patient satisfaction surveys is to understand from the patient perspective a hospital's/institution's or health system in general specific strengths and weaknesses in order to improve the delivery of care (3).

Patient satisfactions involve multidimensional measures to provide further detail within distinct domains, such as interpersonal manner (communication skill and others) of the provider, technical quality of care, availability, outcomes of care and the physical environment (3). Interpersonal manner, technical quality, accessibility/convenience, and finances have been by far the most commonly measured features of care in patient satisfaction studies (5).

Hospitals with high patient satisfaction provided more efficient care with shorter lengths of stay for surgical patients. These hospitals also had higher surgical process quality, lower surgical readmission rates, and lower surgical mortality rates (6).

Not surprisingly, the improvement in lower urinary tract symptoms is mirrored in the quality of life and patient global impression of improvement scores (7). Most men initially reported that they were "mostly dissatisfied" with their symptoms but this improved, and, at followup, they regarded themselves as "pleased" to "mostly satisfied". Most men who were asked to compare their current situation to that before the operation, answered "very much better." No man recorded an unchanged or worse score (7).

Generally, the aim of the treatment of BPH is improving the quality of life (9). R.P. Macdonagh et.al investigated the performance EuroQol (EQ) quality of life measure. The Nottingham Health profile assessed the outcome of TURP for Lower Urinary Tract Symptoms (LUTS) from BPH said that at 12 months after surgery. There were significant improvements in the domains of social interaction, energy, pain, emotional reactions and sleep. The EQ profile also showed a significant improvement in usual activities, mood, and pain/discomfort (9).

Both TURP and open surgery have high profile of efficacy, with marked improvement in International A study done in Japan showed that 
long-term, more than 10 years, symptomatic improvement was sustained with some deterioration, but quality of life (QoL) remained high (10).

TURP is not only proved to be clinically effective, but also improved patients' QoL and bother symptoms. This was associated with longterm, high patient-rated satisfaction (14).

\section{METHODS}

The study design was prospective cohort study. The data was collected at three instances i.e. while the patient was in the ward, at first month, and at third month of the surgery. We took all consecutive patients who had prostatectomy at Menilik II Referral Hospital and Tikur Anbessa Specialized Hospital (TASH) from August 1/2017 to June $15 / 2018$ in Addis Ababa. Convenient sampling technique was used.

All patients who had undergone prostatectomy (either TURP or Transvesical Prostatectomy (TVP)) for BPH by consultant urologist or under the urologist's supervision were included.

Standardized questionnaire was prepared in English and then translated into Amharic and pretest was done. This questionnaire is a standardized tool, and it was developed by the Consumer Assessment of Healthcare Provider and Systems (CAHPS) Clinical and Group Survey.

Participation on the study was only on voluntary basis. We got informed consent from every patient. We also obtained ethical clearance from Surgical Department Research and Publication Committee of Addis Ababa University, College of Health Sciences (AAUCHS).

\section{RESULTS}

During the study period, a total of 103 patients had prostatectomy for BPH, 58 from TASH and 45 from Menilik II Hospital. Based on the exclusion criteria, 5 patients (3 for suspicion and/or diagnosis of prostatic cancer, 1 for presence of concomitant bladder tumor, and 1 patient for having contracted bladder) were excluded. There were two referred patients for pneumonia and Acute Respiratory Distress Syndrome (ARDS) requiring mechanical ventilator. There were also two deaths, and 5 patients were lost from follow-up. Finally, we enrolled 89 patients for subsequent assessment of the level of satisfaction after the procedures (56.2\% TASH, $\quad 43.8 \% \quad$ Menilik). Their demographic data is provided in Table 1. The mean age of the patients was 64.8 (ranging from 45 to 92 ).

Table 1: Demographic data of study participants

\begin{tabular}{lcc}
\hline Variable & Frequency & Percent \\
\hline Age & & \\
45-65 & 54 & 60.7 \\
$65-85$ & 32 & 35.9 \\
Above 85 & 3 & 3.4 \\
Marital Status & & \\
$\quad$ Married & 70 & 78.7 \\
Divorced & 4 & 4.5 \\
Widowed & 12 & 13.5 \\
Single & 2 & 2.3 \\
Not registered & 1 & 1.1 \\
Education & & \\
Illiterate & 19 & 21.3 \\
Able to read and write & 13 & 14.6 \\
Primary education & 17 & 19.1 \\
Secondary education & 16 & 18.0 \\
Diploma and above & 24 & 27.0 \\
Employment & & \\
Employed & 29 & 32.6 \\
Private business & 9 & 10.1 \\
Farmer & 20 & 22.5 \\
Pensioned & 28 & 31.5 \\
Other & 3 & 3.3 \\
\hline
\end{tabular}

When we see the presentation of symptoms of the patients, more than $90 \%$ of them had multiple lower urinary tract symptoms. History of acute urinary retention was present in 50 patients $(60.2 \%)$; and 54 patients $(62.8 \%)$ had history of catheterization. More than half of these patients stayed with symptoms for more than 1 year before the surgery.

Fifty-eight $(65.2 \%)$ of the patients had TURP, and the rest had TVP. 
Table 2: Preoperative clinical finding

\begin{tabular}{lll}
\hline Symptoms & Frequency & Percent \\
\hline Straining at micturition & 87 & 97.7 \\
Poor urinary stream & 84 & 94.3 \\
Urgency & 79 & 88.7 \\
Frequency & 82 & 92.1 \\
Nocturia (3 times and above) & 66 & 74.1 \\
Urinary incontinence & 53 & 60.9 \\
Acute urinary retention & 50 & 60.2 \\
History of catheterization & 54 & 62.8 \\
\hline
\end{tabular}

From all patients who had the above procedures, there were six intraoperative bleeding, five during TVP and 1 during TURP. Three Patients required intraoperative and/or post-anesthesia care unit blood transfusion. The intraoperative TURP bleeding forced the surgeon to stop the procedure.

There were no major postoperative complications except 2 patients who had bleeding which required transfusion in the ward. The minor complications found were clot retention $(n=4)$ and wound site infection $(n=3)$. No patient had TURP syndrome. We had two reoperations, the first was re-exploration who developed significant bleeding after TVP and was done immediately after the primary surgery, and the second was re-TURP which was done for incomplete resection because of bleeding which forced the surgeon to terminate the procedure. There were two deaths; one from each type of the surgery. Two patients were also referred to mechanical ventilator after they developed post-operative pneumonia and ARDS.

The majority of the patients $(n=50,56.1 \%)$ were discharged within three days of the postoperative period, and a total of $93.3 \%$ of the patients were discharged within seven days of the procedure. About eighty three per cent $(84.3 \%)$ of these participants wre very satisfied with the doctors' care. About ten per cent $(10.2 \%)$ and four and half percent $(4.5 \%)$ of the participants were very dissatisfied with the care given by nurses and doctors respectively. The level of patient satisfactions were assessed for urinary and sexual function and are shown in the figure below (Figures 1 and 2).

\section{MEASUREMENT AFTER ONE MONTH POST- SURGERY}

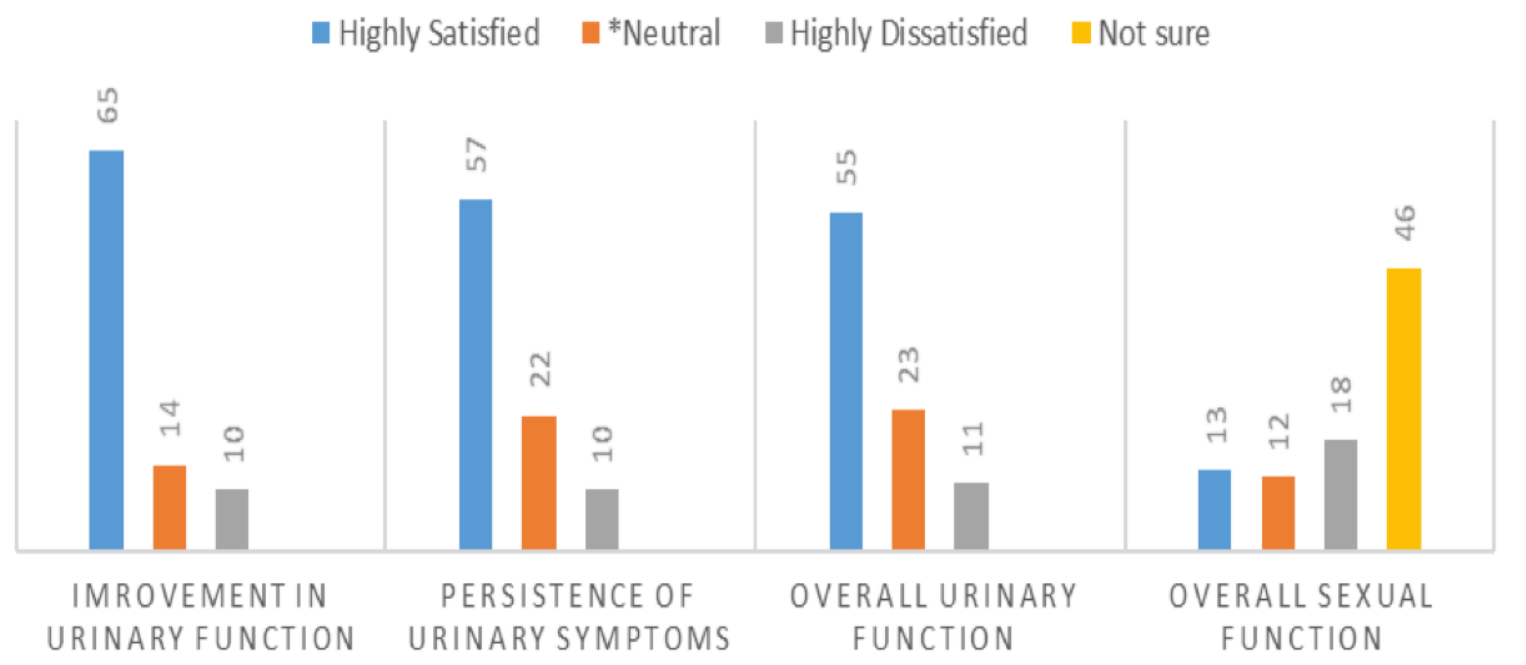

Figure 1: Level of satisfaction from urinary and sexual function after the surgery 


\section{AFTER THIRD MONTH POST-SURGERY}

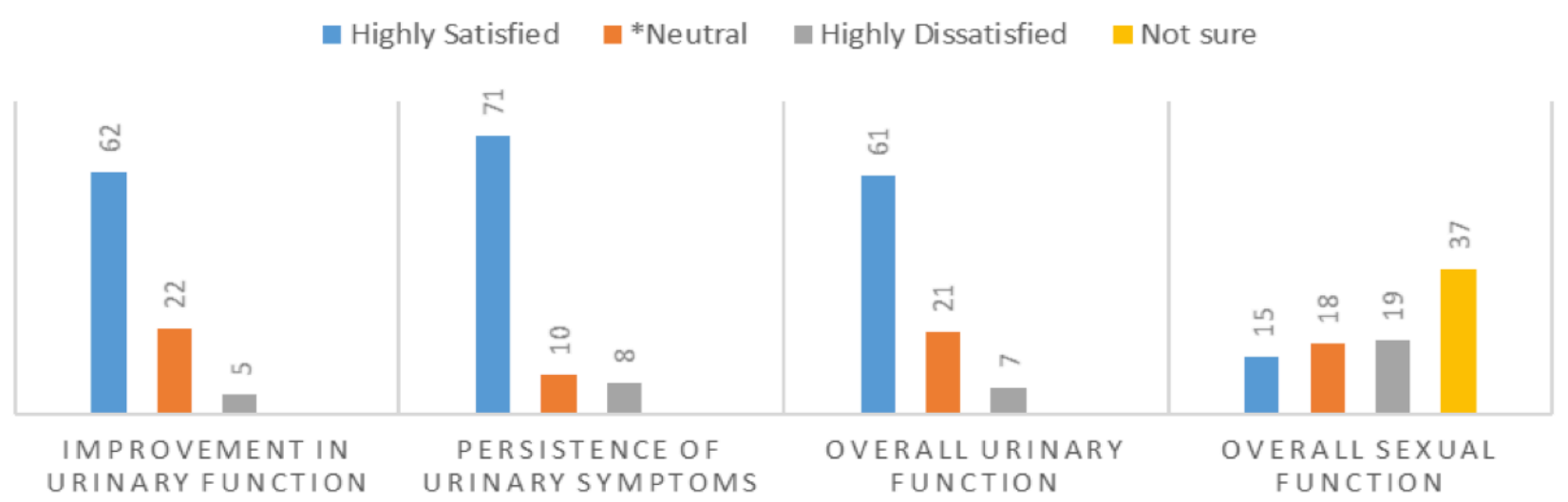

Figure 2: Level of satisfaction from urinary and sexual function after the surgery

In addition to the assessment of urinary and sexual function, patients were also inquired whether they experienced informed or uninformed urinary, sexual, and general surgical complications. The data showed at the end of the first month of the surgery, $54 \%$ and $13.6 \%$ of the patients had urinary and sexual complications about which they were informed, respectively. Those who experienced untold urinary and sexual complications accounted for 16.7 and $14.7 \%$ of the respondents respectively.

For the hospital environment, around $60 \%$ of the patients reported they were very satisfied with cleanliness of the rooms and bath rooms and also the quietness of the rooms at night. On the contrary, around $16 \%$ were very dissatisfied about the cleanliness of the rooms and bathrooms, and $10 \%$ were also very dissatisfied with the quietness of the rooms at night.

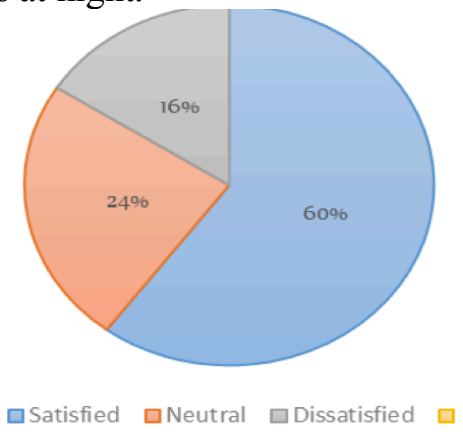

Figure 3: Assessment of satisfaction with cleanliss of rooms and bathrooms
Three key questions were asked about the overall satisfaction of the patients on the care provided and the overall assessment of the hospitals. These questions were whether they recommend the same procedures they have undergone to families and close friends. In addition they were asked if recommend the hospitals to their families and close friends. Finally they were asked to grade the hospitals from 1 to 5 (1 being the worst hospital and 5 being the best hospital).

Table 3: Assessment of overall satisfaction level of participants.

\begin{tabular}{lll}
\hline Variable & Frequency & Per cent \\
\hline $\begin{array}{l}\text { Recommend the } \\
\text { procedure: }\end{array}$ & & \\
$\quad$ Yes & 78 & 87.6 \\
$\quad$ No & 11 & 12.4 \\
$\begin{array}{l}\text { Recommend the } \\
\text { hospital: }\end{array}$ & & \\
$\quad$ Yes & 56 & 62.9 \\
$\quad \begin{array}{l}\text { Neutral } \\
\quad \text { No }\end{array}$ & 24 & 27.0 \\
$\begin{array}{l}\text { Rank given to the } \\
\text { hospital: }\end{array}$ & 9 & 10.1 \\
$\begin{array}{l}\text { Among the good } \\
\text { hospitals }\end{array}$ & 59 & 66.3 \\
$\begin{array}{l}\text { Neutral } \\
\text { Among the }\end{array}$ & 21 & 23.6 \\
bad/poor hospitals & 9 & 10.1 \\
\hline
\end{tabular}

DOI: http://dx.doi.org/10.4314/ejhs.v30i5.12 


\section{DISCUSSION}

Surgical management of enlarged prostate with lower urinary tract symptoms has different modalities; however, TURP is still considered as the 'gold standard'.

All of our patients received prophylactic antibiotics. The only significant intraoperative and perioperative complication we found was bleeding requiring transfusion; around $7 \%$ of the patients who had TVP had significant bleeding requiring transfusion while none of the patients who had TURP needed blood transfusion. These results may lead to the conclusion that both procedures are safe procedures. This low incidence of complications is even lower than most of the studies reviewed $(7,10,11,15)$.

Satisfaction from surgical outcome of the urinary function is reasonably high and also seems to improve with time as the relative number of patients who were highly satisfied in the first month is increased during third month measurement (Figures 1 and 2). Participants who were dissatisfied with their sexual outcome (from those who had sexual intercourse) were found to be high (around 54.5\% at first month and 20\% at third month). Most (90.7\%) of the patients asked at third month of the post-surgery reported that they recommend the same surgery they had undergone, which can lead us to conclude that both procedures have high degree of satisfaction. With regard to satisfaction level about hospital environment, around two-thirds of the patients were satisfied with the hospitals.

When we consider recommending the surgery, recommending the hospital and rank given to the hospital as a proxy indicators of satisfaction, our results showed outcome related to urinary function and communication skills of healthcare providers to higher relationship.. There was also relationship between level of satisfaction and hospital stay; hospital stay of more than 7 days is shown to be associated with patient dissatisfaction.

Sexual outcome had low relation with the satisfaction with the surgery, and type of surgery was not shown to be related to level of satisfaction. To conclude, whether rate of complications, rate of reoperations, and subsequent procedures related to prostatectomy affect level patient satisfaction or not was difficult because of low incidence of these events.

In conclusion, both TURP and open prostatectomy had high level of patient satisfaction and are also related to significant level of subjective urinary function improvement and have relatively good safety profile. Thus, we recommend both procedures for patients with $\mathrm{BPH}$ who are candidates for surgical treatment. Satisfaction about the hospitals was also found to be relatively high.

As both procedures had high level of patient satisfaction and safety profile, more urologists need to be trained in both procedures. The facility for endourology should be available in different parts of the country. General surgery residents should should be well trained with skills in open prostatectomy.

\section{REFERENCES}

1. J. de la Rosette, G. Alivizatos, S. Madersbacher,C. Rioja Sanz, J. Nordling, M. Emberton, Guidelines on Benign Prostatic Hyperplasia, European Association of Urology. 2002 Feb, pp5

2. American Urological Association treatment guideline; management of benign prostatic hyperplasia, revised 2010

3. Eric Shirley, Gary Josephson, James Sanders, Fundamentals of Patient Satisfaction measurement, Physician Leadership Journal. 2016 Jan/Feb; 3(1):12-17.

4. LaVela, Sherri L, Evaluation and measurement of patient experience, Patient Experience Journal. 2014 April; 1(1); 28-36.

5. John E. Ware JR, Defining and Measuring Patient Satisfaction With Medical Care: Evaluation and Program Planning. 1983; 6(3-4):247-263.

6. Thomas C. Tsai, Orav EJ, Jha AK. Patient Satisfaction and Quality of Surgical Care in US Hospitals, Ann Surg. 2015 Jan;261(1):2-8.

7. Rafael Marmiroli, Alberto A. Antunes, Sabrina Reis, Elcio Nakano, Miguel Srougi, Standard surgical treatment for benign prostatic hyperplasia is safe for patients over 75 years: Analysis of 100 cases from a high-volume urologic center, Clinics 2012;67(12):1415-1418.

8. Mishriki SF, Grimsley SJ, Nabi G, Martindale A, Cohen NP Improved quality of life and enhanced satisfaction after TURP: prospective 12-year follow-up study, Urology. 2008 Aug;72(2):322-6

9. Arie Carneiro, Paulo Sakuramoto, Marcelo Langer Wroclawski, et al. Open suprapubic versus retropubic prostatectomy in the treatment of benign prostatic hyperplasia during resident's learning curve: a randomized controlled trial, Interational Brazil Journal of Urology. 2016: 42(2):284-292.

10. Masumori N1, Furuya R, Tanaka Y, Furuya S, Ogura H, Tsukamoto T. The 12-year symptomatic outcome of transurethral resection of the prostate for patients with lower urinary tract symptoms suggestive of benign prostatic obstruction compared to the urodynamic findings before surgery, BJU Int. 2010 May;105(10):1429-33. 